\title{
Research on Human Vehicle Accident Reconstruction Based on AEB System
}

\author{
Yican Zhu \\ Shanghai University of Engineering Science, SUES, Shanghai, China \\ Email: 596696484@qq.com
}

How to cite this paper: Zhu, Y.C. (2018) Research on Human Vehicle Accident Reconstruction Based on AEB System. Open Access Library Journal, 5: e4956. https://doi.org/10.4236/oalib.1104956

Received: October 2, 2018

Accepted: October 26, 2018

Published: October 29, 2018

Copyright (๑) 2018 by author and Open Access Library Inc.

This work is licensed under the Creative Commons Attribution International License (CC BY 4.0).

http://creativecommons.org/licenses/by/4.0/

\section{(c) (i) Open Access}

\begin{abstract}
Accident reconstruction refers to the physical activity of inferring the accident process according to the traces left by the accident participants, the final position, the participation in the car damage and the injury of the participants, and then the testimony of the participants and eyewitnesses. It not only provides a basis for the identification of the faults of both parties, but also provides data support for accident prevention and vehicle active safety analysis and research. This paper studies the basic theory of accident reconstruction, and uses PC-Crash software to reconstruct 30 accidents, and obtain parameters such as vehicle, human motion parameters and relative position before, during and after collision.
\end{abstract}

\section{Subject Areas}

Mechanical Engineering

\section{Keywords}

Car Accident, Accident Reconstruction, Active Safety, AEB System

\section{Introduction}

\section{Selection of People's Car Accident Samples}

The purpose of accident reconstruction in this paper is to obtain parameters such as the kinematic parameters related to people and vehicles before the accident, and provide data for the evaluation of the pedestrian AEB system. Taking the typical vehicle crash accident conditions as the basic criteria, the pedestrian AEB system is used to configure the vehicle to select the accident [1]. The following conditions are met for the collision of people and vehicles:

1) The accident vehicle models are cars, SUVs, MPVs, of which cars are mainly used; 
2) The selected accidents are collisions with pedestrians at the front of the vehicle, and no pedestrian crushing occurs during the collision;

3) In each case of pedestrian accident, the pedestrian participation is one person and the pedestrian is an adult.

In the end, 30 cases of human-car collision accidents were selected, including 19 cases of death accidents. The biggest injury to the injured pedestrians was MAIS3+ [2]. The most serious injuries in the 11 pedestrians were AIS3+.

\section{The Theoretical Basis of PC-Crash Software and Its Vehicle Accident Reconstruction}

\subsection{PC-Crash Software Introduction}

The PC-Crash software is an accident reconstruction software developed by $\mathrm{H}$. Steffan et al. of the University of Graz, Austria, based on the Kudfich-Slibar model. It can perform collision analysis such as automobile and pedestrian. The theoretical basis of PC-Crash software is kinematics theory and dynamics theory. Based on the principles of momentum conservation and energy conservation, the relevant equations of the accident participants are established. The final position of the accident participants is used to estimate the motion process before the collision (Figure 1).

Through the information of the ground tire traces at the accident site, the final position of the participants, the damaged parts of the vehicles and the damage parts of the pedestrians, the accident collision process is optimized, and the accident process that meets the information of the legacy of the accident is finally obtained. The reproduction results are shown in an animated form, which is more intuitive and easy to understand. Information such as the exercise parameters

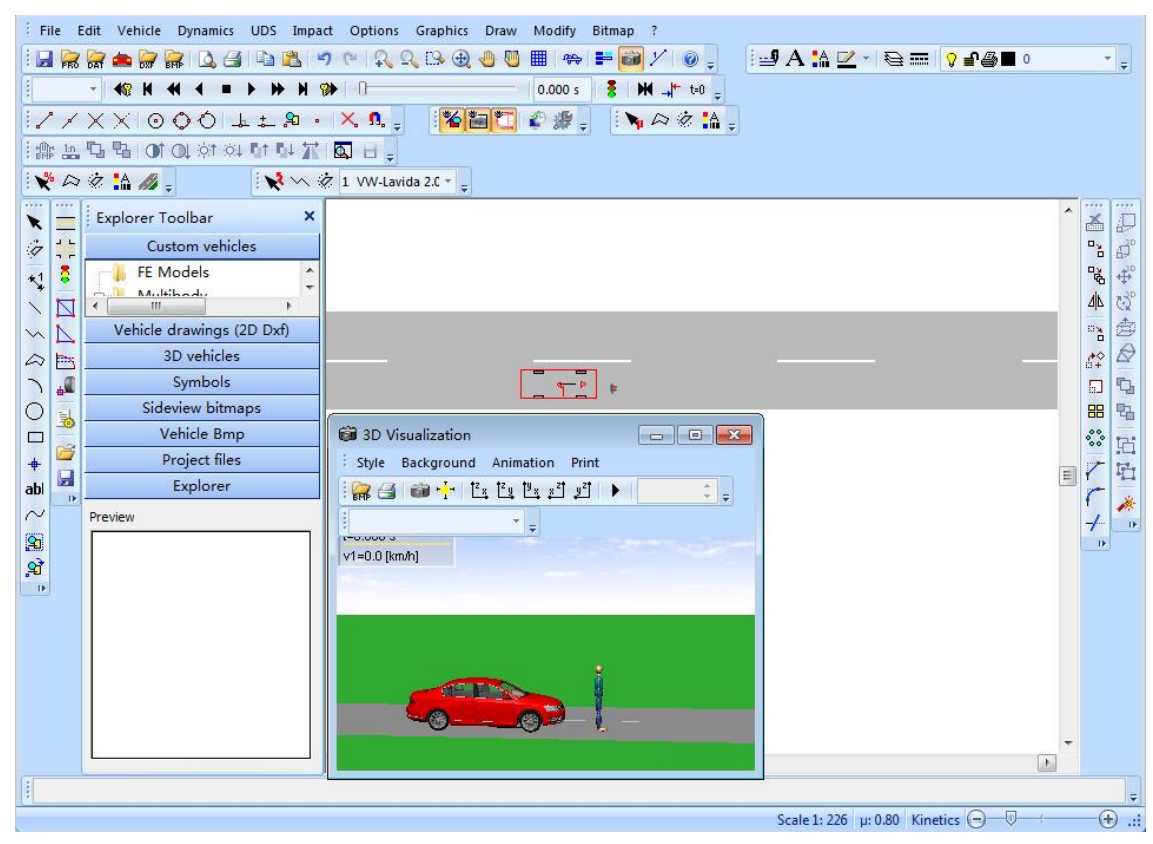

Figure 1. Interface of PC-Crash software. 
and driver's operation status of each participant obtained by the accident reconstruction can be read.

\subsection{Vehicle Model}

In PC-Crash, to simplify the definition of vehicle geometry model, three coordinate systems, global coordinates OXYZ, vehicle coordinates and tire coordinates are introduced, as shown in Figure 2. The specific definition is as follows.

The global coordinate center is the center of the interface initialized by the Pc-Crash software, and the plane is the ground. The $\mathrm{X}$ axis points to the right side of the computer interface, the $Y$ axis points to the upper part of the computer interface, and the $\mathrm{Z}$ axis is in line with the direction of gravity acceleration [3]. The center of the vehicle coordinates is the position of the center of gravity of the vehicle. The $\mathrm{X}$ ' axis points to the front of the vehicle along the longitudinal axis of the vehicle. The axis points to the upper part of the computer interface, and the axis is in the same direction as the acceleration of gravity. The center of the tire coordinate is the contact point between the tire and the ground. The plane is the ground. The axis points to the front of the vehicle along the longitudinal axis of the tire. The axis points to the left side of the wheel, and the axis points vertically to the upper part.

In the inertial coordinates, the position of the center of gravity of the vehicle is defined by an appropriate amount. The rotational motion of the vehicle is synthesized by three independent rotational motions, representing the rotation, pitch and roll motion of the vehicle. The three independent rotational motions are the rotational motion of the axis, the rotational motion of the axis, and the rotational motion of the axis. As shown in Figure 3.

The vehicle is subjected to external forces such as tire force, air resistance, gravity and rigid body interaction.

\subsection{Multi-Rigid Pedestrian Model}

For the real simulation of pedestrian dynamics in the collision process, the

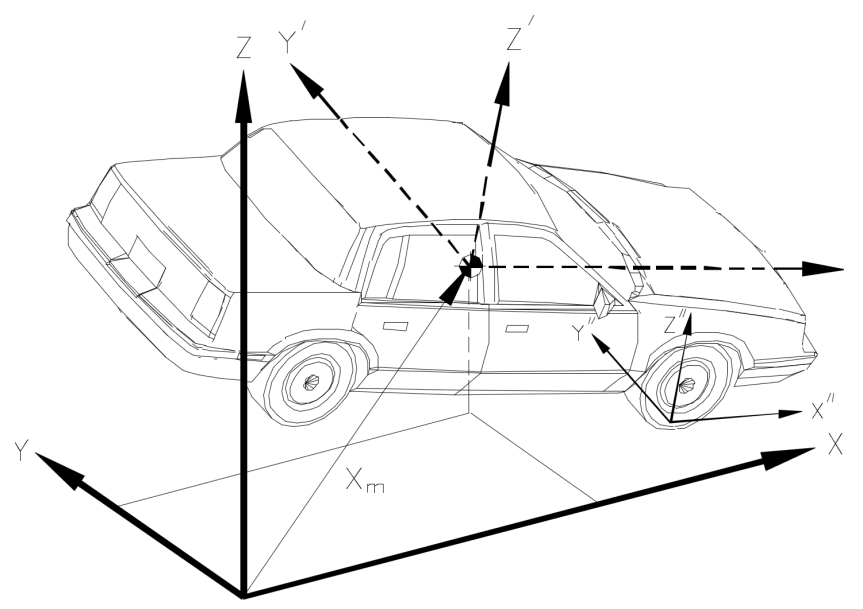

Figure 2. Car coordinates diagram. 


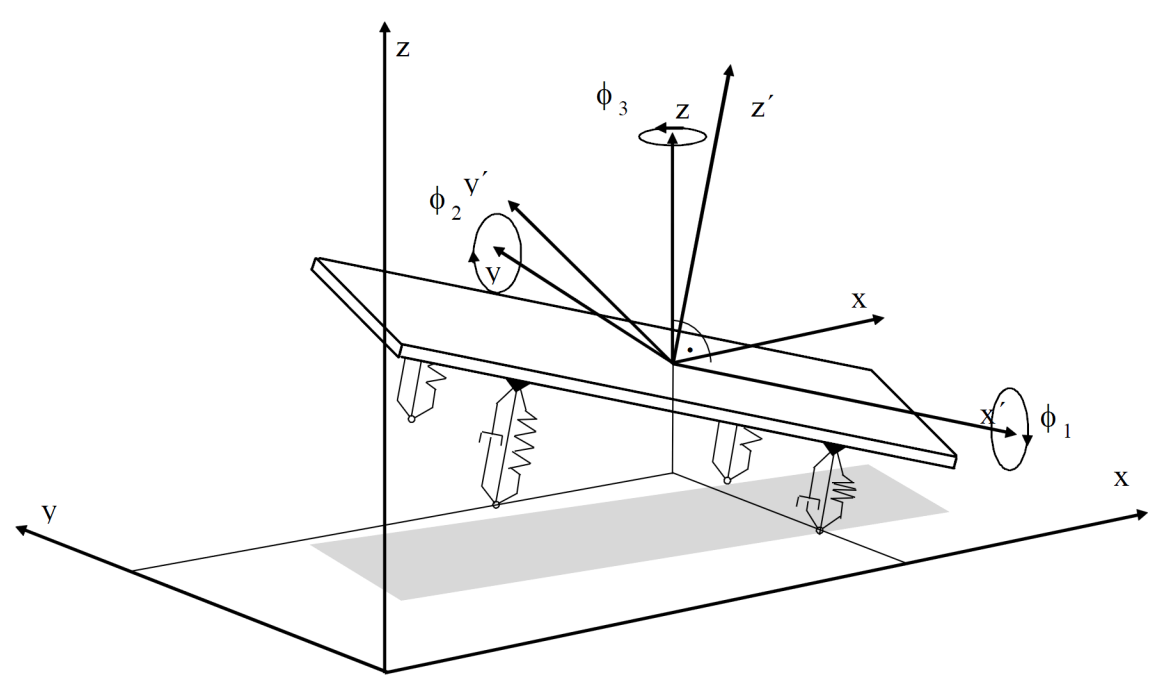

Figure 3. Rotational movement of the car.

pedestrian model is simulated by a multi-rigid system, and the pedestrian rigid body is connected by a rigid body and a hinge. The multi-rigid pedestrian model not only facilitates the confirmation of the collision area between the vehicle and the pedestrian, but also facilitates the setting of the pedestrian height, weight and other characteristics and the pedestrian collision movement state. The pedestrian rigid body model is shown in Figure 4.

1) Pedestrians with multiple rigid body coordinates

The inertial coordinate system used in the pedestrian multi-rigid dummy model and the two coordinate systems of the ellipsoid carrier coordinate system fixed to the rigid body itself are shown in Figure 5. The coordinate axis direction of the ellipsoid carrier coordinate system is determined by the ellipsoidal half-axis direction. Both coordinates are right-handed coordinate systems.

2) Pedestrian multiple rigid body motion equation

When the multibody system is calculated, the external forces (gravity, contact force, friction, and force between the joints) of the dummy's body are calculated in each time step [4]. In addition to the external forces received by the dummy, the solution of the established mathematical equation determines the motion state of the multi-rigid body.

The equation of motion based on the principle of conservation of angular momentum and the principle of force balance is used to describe the motion response of each part of the body of the dummy under the action of external force and momentum.

$$
\begin{gathered}
m_{i} \cdot \vec{x}=\sum_{j} \vec{F} \\
F=\left(\begin{array}{ccc}
I_{x x} & -I_{x y} & -I_{x z} \\
-I_{x y} & I_{y y} & -I_{y z} \\
-I_{x z} & -I_{y z} & I_{z z}
\end{array}\right)
\end{gathered}
$$

3) Definition of joints 


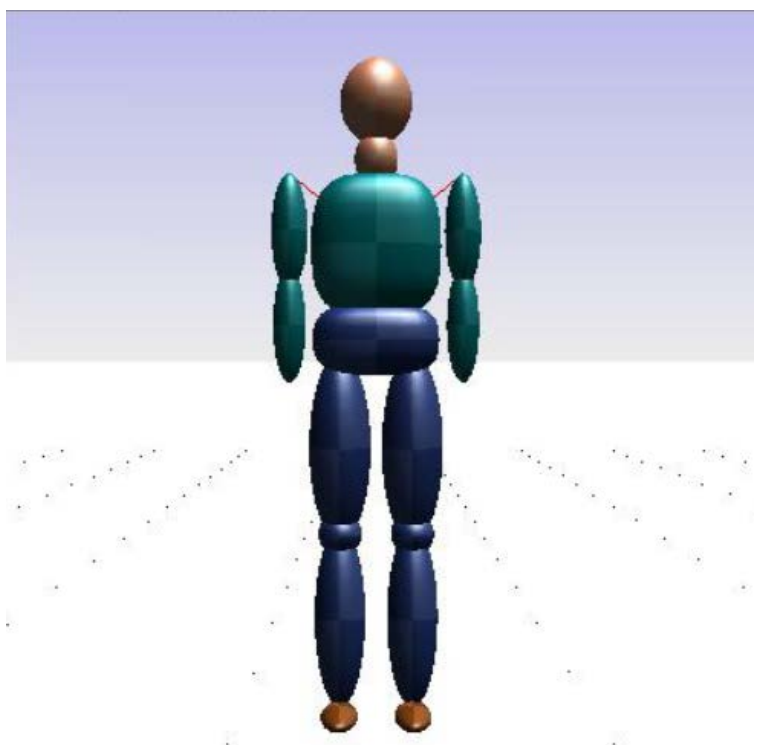

Figure 4. Pedestrian model.

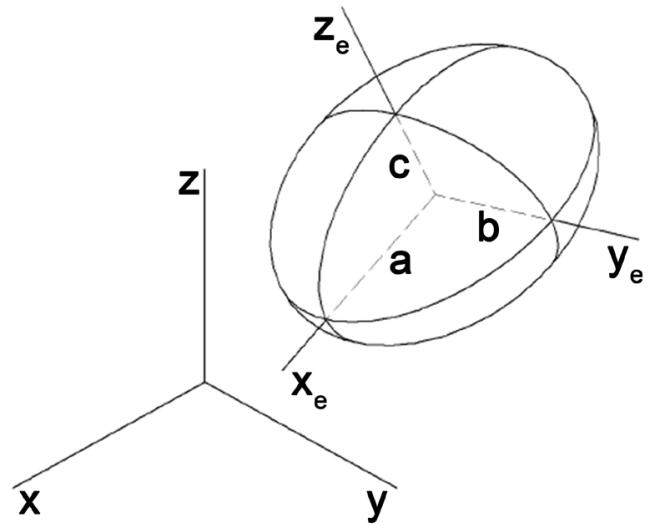

Figure 5. The inertial coordinate system and carrier coordinate system.

The relative movement between the two adjacent rigid bodies is generally constrained by a hinge. Each joint is used to connect two parts and defines the position of the joint and some parameters that describe the joint properties. The joint direction vector and, are defined in the rigid body local coordinate system connected to the joint. The movement of the rigid body around each axis of the joint can be limited by increasing the corresponding rotational stiffness, as shown in Figure 6.

The joint motion equation of the multi-rigid dummy system is numerically solved in each time step. In order to meet the above requirements, there are two assumptions: the strength of the two rigid bodies connected by the joints is equal and opposite in direction; the acceleration of the rigid bodies of the two adjacent body parts at the joint is the same.

The equations of joint motion of multiple rigid bodies are generally solved by the Lagrangian algorithm. First calculate the external force received by the multi-rigid body system, and then consider the friction between the multiple rigid 


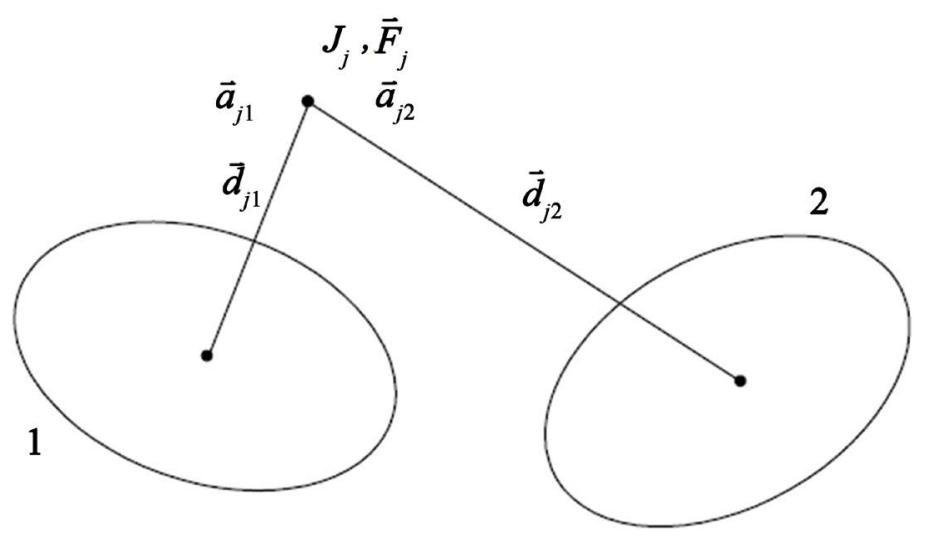

Figure 6. Joint definition.

bodies. The reciprocating iterative algorithm is used in the calculation process until the force at the joint does not change significantly. The geometrical constraints are replaced by the reaction forces on the joints, and each rigid body can be analyzed separately using the isolator method. The disadvantage of this method is that it only guarantees that the accelerations at the joints are equal and cannot guarantee the same speed, so that in a large time step, the two parts of the multi-rigid body may be separated.

\section{Accident Case Reconstruction}

\subsection{Case Introduction}

On a sunny day, the street lights and lights are normally turned on, the traffic volume is normal, and the road surface is dry asphalt road. A Volkswagen LaVida car is driving in the middle straight lane of one-way three lanes. A road maintenance person walks in the opposite direction in the opposite direction for construction inspection. The Langyi driver failed to find the other party in time, and eventually the two collided. In the collision, the pedestrian's thigh collides with the right side of the front bumper skin on the right side of the front bumper. The head collides with the right side wiper, and there are collision marks on the front bumper skin, the hood and the front windshield. There were brake tracks, a pedestrian's shoes and a blood mark on the scene.

\subsection{Accident Site Reconstruction}

The site map drawn according to the on-site information collection is imported into the PC-Crash. After the accident site is imported, the vehicle and ground friction coefficient needs to be set according to the weather conditions and road conditions. In addition, according to the actual road conditions, the road surface slope and road surface height are determined. Wait for the corresponding adjustments.

\subsection{Establishment of Accident Vehicle Model}

The vehicle model can be called from the PC-Crash vehicle model library. 
PC-Crash has a rich vehicle model database. The data can include cars, trucks, trailers, motorcycles, etc. In the accident reconstruction, it can be called directly and according to the actual situation. Make adjustments in the vehicle settings. The front structure of the vehicle and the position of the center of gravity of the vehicle that have a great influence on the collision result in the vehicle accident should be checked and adjusted according to the actual collected data. According to the car parameters involved in the accident, the model of the car is built in PC-Crash, as shown in Figure 7.

\subsection{Pedestrian Model}

In this accident, the pedestrian was $1.66 \mathrm{~m}$ tall and weighed $70 \mathrm{~kg}$. The pedestrian model in PC-Crash is divided into single rigid body pedestrian model and multi-rigid pedestrian model. Single rigid body pedestrian model is used for pedestrian motion simulation before collision Multi-rigid pedestrian model is used to simulate pedestrian dynamic response during collision [5]. For the multi-rigid pedestrian model, the response should be set according to the pedestrian parameters in the accident. The multi-rigid pedestrian model after setting the relevant parameters is shown in Figure 8.

In addition, the state of motion of pedestrians has a greater impact on the results of reconstruction. Therefore, in the reconstruction, it is necessary to refer to the transcripts of the driver and the eyewitness and the related information of the pedestrian's injury site to determine the state of motion of the pedestrian during the collision. In the multi-rigid pedestrian model, the movement of the dummy's head, torso, thigh, calf, etc. can be used to simulate different movement states of the pedestrian. The state of pedestrian standing, walking, jogging, running, etc. is shown in Figure 9.

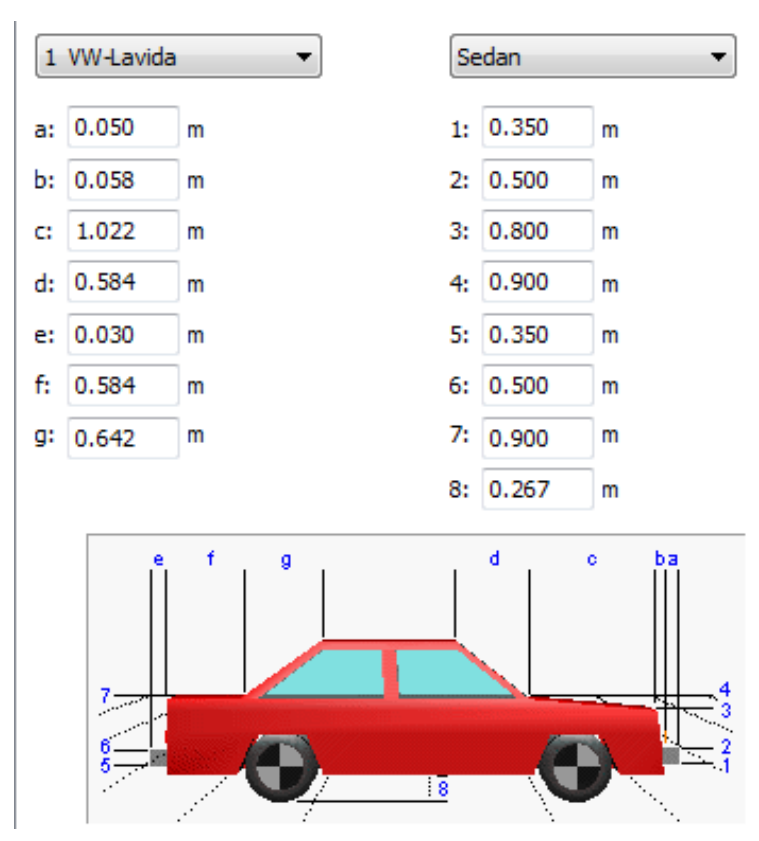

Figure 7. Car model. 


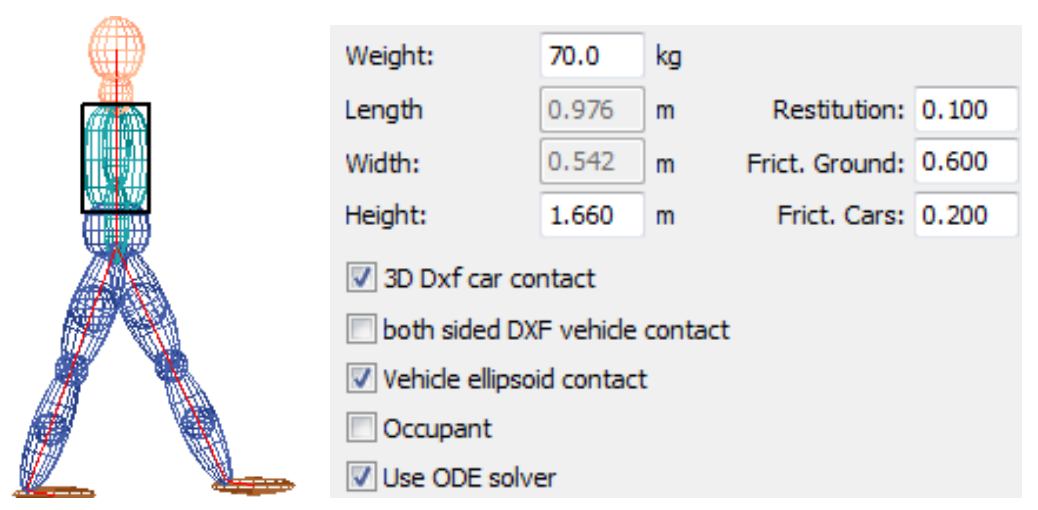

Figure 8. Pedestrian model.

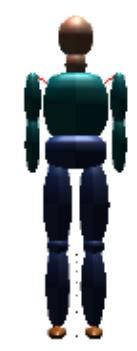

(a)

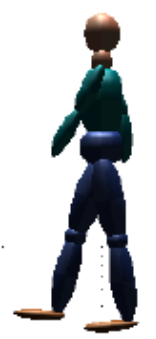

(b)

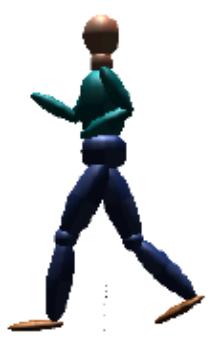

(c)

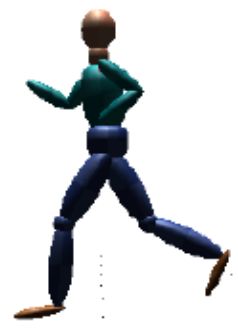

(d)

Figure 9. Pedestrian model in different motion states. (a) Stand; (b) Walk; (c) Jog; (d) Run.

\subsection{Parameter Selection}

1) Vehicle collision speed

In the PC-Crash reconstruction, the collision instant to the end of the collision process is first simulated. In this process, the PC-Crash calculation mode is first changed to the dynamic mode. For the collision speed of cars and pedestrians, the pre-estimation of the collision speed should be based on the road marking method, the pedestrian throwing method and the video method. The vehicle braking and steering are set according to the road surface traces, the scattered objects on the scene, and the related witnesses' transcripts. The vehicle related parameter settings in this accident are shown in Figure 10.

2) Pedestrian motion parameters before collision

According to the age, gender and eyewitness description of pedestrian movement, the pre-collision motion parameters are set as shown in Figure 11.

\subsection{Reconstruction Process and Results Verification}

1) Accident reconstruction and verification after collision and collision

This reconstruction process starts from the first collision point of the vehicle and the pedestrian, and continuously adjusts the relevant parameters to optimize the simulation results, and finally obtains the results in line with the actual situation.

The resulting vehicle and pedestrian movement process is as shown in Figure 12. The final position of the vehicle and pedestrian simulation is basically consistent 


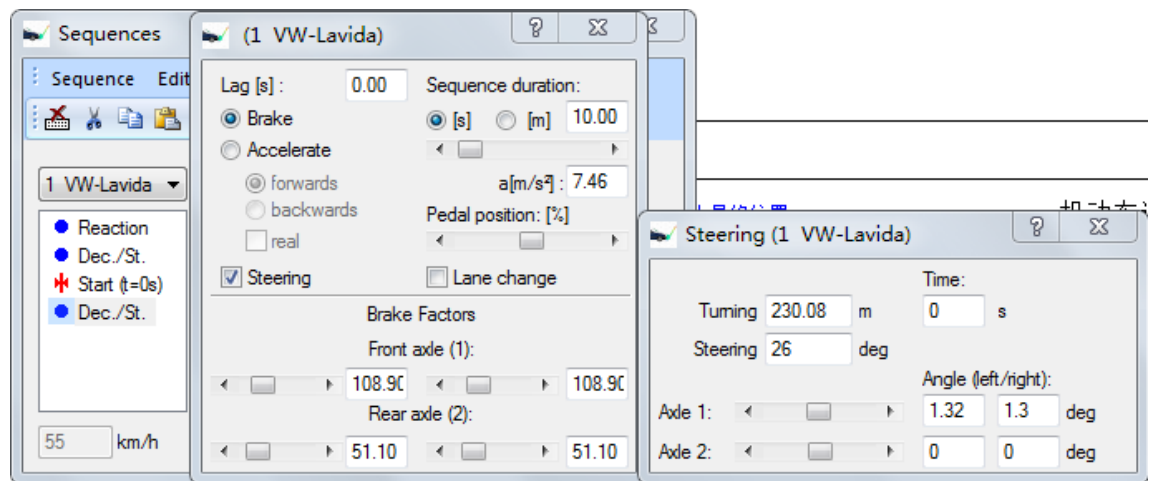

Figure 10. Motion parameters of Lavida after collision.

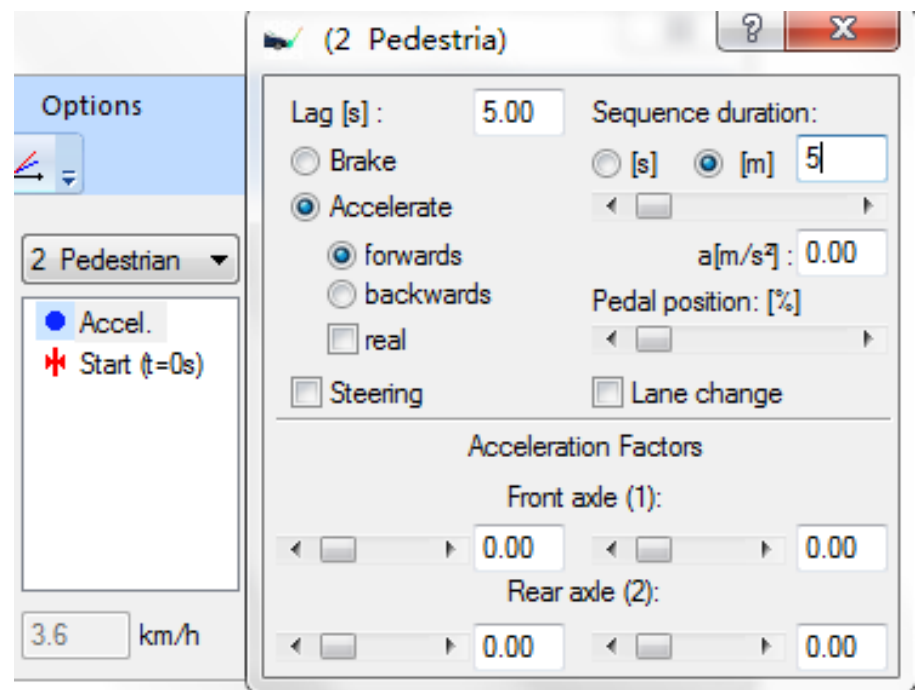

Figure 11. Movement parameters of pedestrian before collision.

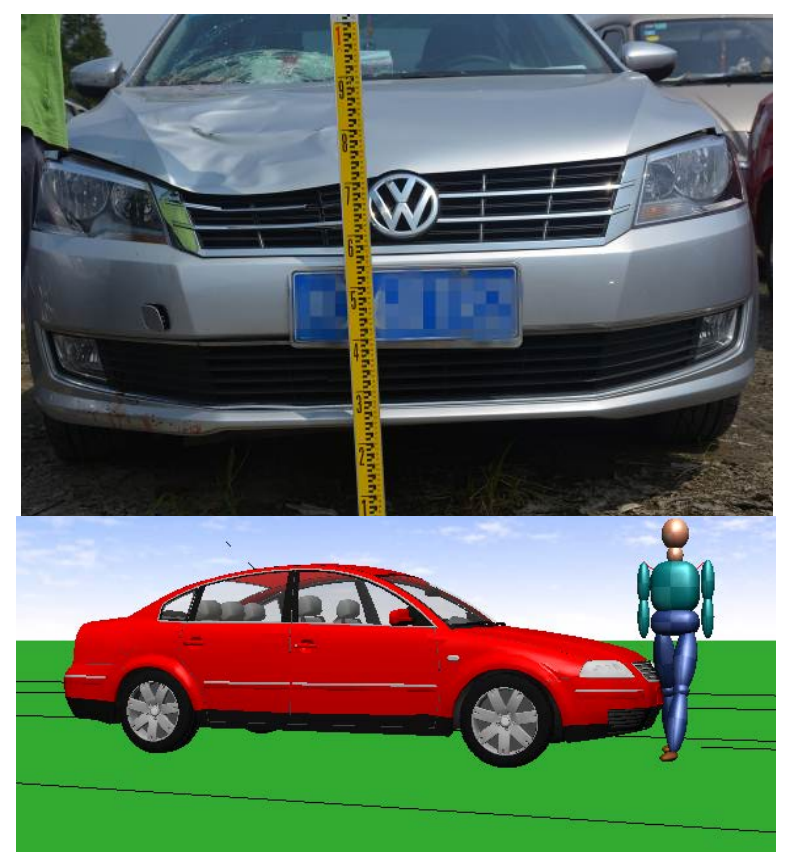

Figure 12. Correspondence between pedestrian's legs of the car collision. 
with the final position of the acquisition, and the left wheel of the vehicle moves along the ground brake marks, and the pedestrian head tribe slips in line with the ground blood mark.

The collision position between the pedestrian model's leg and the front bumper skin is consistent with the vehicle's damaged position, and the pedestrian collision position is consistent with the pedestrian injury (Figure 13).

The pedestrian model hip and hood collision position is consistent with the vehicle damage position, and the pedestrian collision position is consistent with the pedestrian injury (Figure 14).

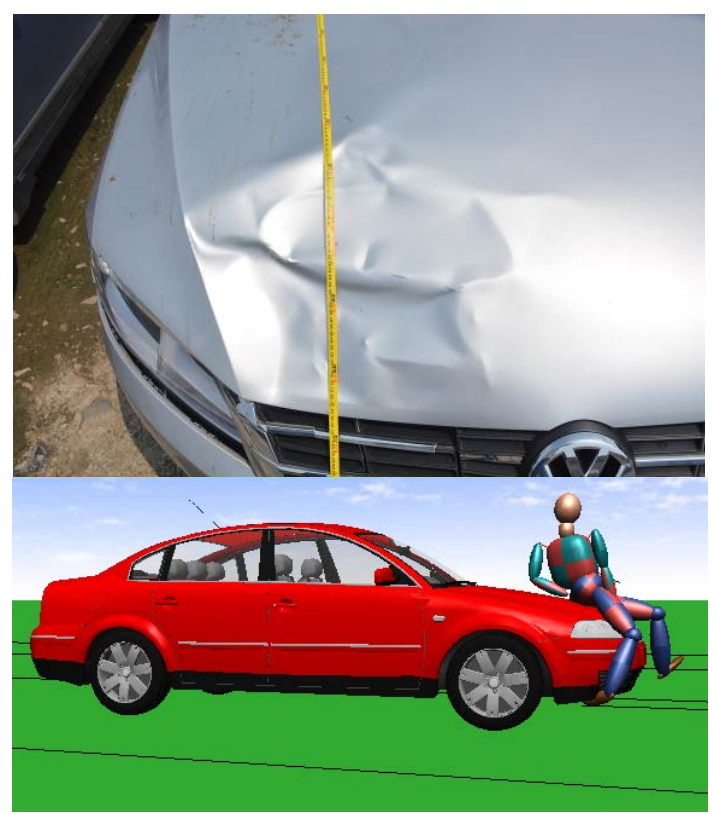

Figure 13. Correspondence between pedestrian's hip and car collision.

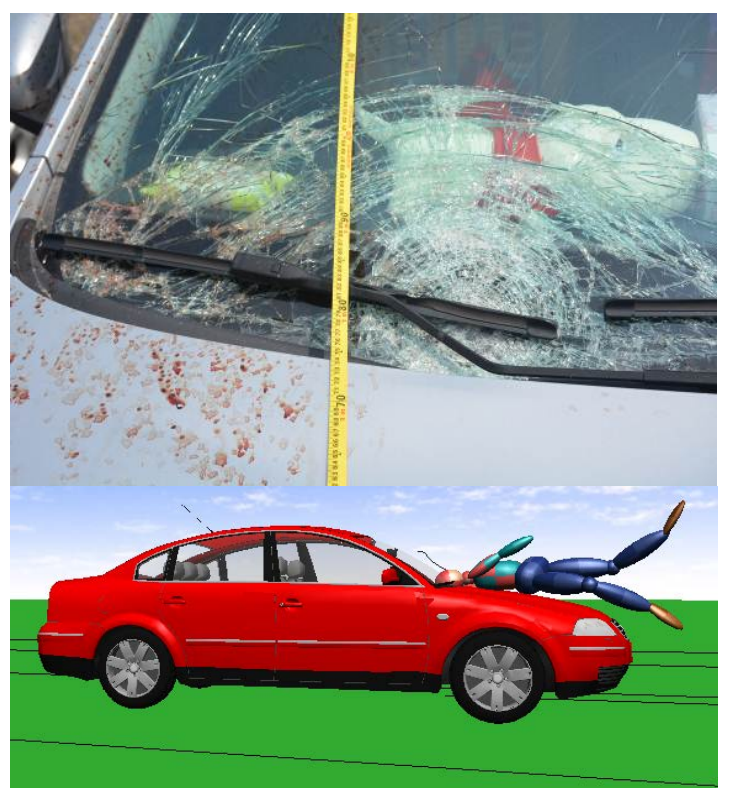

Figure 14. Correspondence between pedestrian's head and the car collision. 
The pedestrian model head and hood collision position are consistent with the vehicle damage position, and the pedestrian collision position is consistent with the pedestrian injury.

2) Accident reconstruction before collision

Different from the accident collision process, the two participants have no mutual force before the collision, so the simulation can adopt the kinematics mode. According to the existing AEB system startup time [6], the process of $5 \mathrm{~s}$ before the collision is reconstructed, and the obtained data can satisfy the evaluation of the AEB system. The vehicle and pedestrian movement status before the accident is set by the driver's transcript. For the case where the pedestrian is covered by the occluded object, the motion simulation before the pedestrian collision is simulated from the moment when the pedestrian walks out of the occlusion. The resulting reconstruction results, the entire accident process is consistent with the collected accident-related information.

\section{Conclusion}

In this paper, the accident reconstruction theory and reconstruction method based on PC-Crash software are studied, and the accident reconstruction is carried out by PC-Crash. The vehicle and pedestrian motion parameters and the relative positions of the pedestrians are obtained $5 \mathrm{~s}$ before the collision. It is the preparation for accident reconstruction. The accident reconstruction data are an extension and supplement to the collected data. This provides useful data for the establishment of the pre-accident dangerous data matrix and provides basic data support for the development of active safety systems.

\section{Conflicts of Interest}

The authors declare no conflicts of interest regarding the publication of this paper.

\section{References}

[1] Lawrence, G.J.L., Hardy, B.J., Carroll, J.A., et al. (2004) A Study on the Feasibility of Measures Relating to the Protection of Pedestrians and Other Vulnerable Road Users. Transportation Research Library.

[2] McLaughlin, S.B., Hankey, J.M. and Dingus, T.A. (2008) A Method for Evaluating Collision Avoidance Systems Using Naturalistic Driving Data. Accident Analysis \& Prevention, 40, 8-16. https://doi.org/10.1016/j.aap.2007.03.016

[3] Huang, S., Yang, J. and Eklund, F. (2008) Evaluation of Remote Pedestrian Sensor System Based on the Analysis of Car-Pedestrian Accident Scenarios. Safety Science, 46, 1345-1355. https://doi.org/10.1016/j.ssci.2007.08.004

[4] Rosén, E., Källhammer, J.E., Eriksson, D., et al. (2010) Pedestrian Injury Mitigation by Autonomous Braking. Accident Analysis \& Prevention, 42, 1949-1957. https://doi.org/10.1016/j.aap.2010.05.018

[5] Fredriksson, R. and Rosen, E. (2012) Integrated Pedestrian Countermeasures-Potential of Head Injury Reduction Combining Passive and Active Counter 
Measures. Safety Science, 50, 400-407. https://doi.org/10.1016/j.ssci.2011.09.019

[6] Anderson, R., Doecke, S., Mackenzie, J., et al. (2013) Potential Benefits of Autonomous Emergency Braking Based on In-Depth Crash Reconstruction and Simulation. 23rd International Technical Conference on the Enhanced Safety of Vehicles, 27-30. 